\title{
Theoretical considerations concerning the profile error of the thread flank
}

\author{
Laurențiu Slătineanu', Oana Dodun ${ }^{1, *}$, Irina Beșliu Băncescu², Ionel Coman ${ }^{1}$, Adrian \\ Ghionea $^{3}$ and Traian Mihordea ${ }^{4}$ \\ 1 “Gheorghe Asachi” Technical University of Iași, Blvd. 59A, 700050 Iași, Romania \\ 2 "Ștefan cel Mare" University of Suceava, Universităţii Street, 13, 720229 Suceava, Romania \\ ${ }^{3}$ Politehnica University of Bucharest, Splaiul Independenței 313, 060042 Bucharest, Romania \\ ${ }^{4}$ Romanian Welding Society, Iași County Branch, Vasile Lupu Street, 85, Iași, Romania
}

\begin{abstract}
In the case of metric thread, a possible source of the flank error could be the position of the cutting tool edge, which could not intersect the circular cylindrical surface axis. An analytical method of approximation was applied to model the way in which the flank error is generated by considering some geometrical conditions. A theoretical simplified model was determined to highlight the influence of the thread external diameter and of the distance between the circular cylindrical surface axis and the thread rectilinear generatrix on the profile error of thread flank.
\end{abstract}

\section{Introduction}

The threaded surfaces are surfaces obtained by moving a certain plane profile along a spiral. These surfaces are applied in the case of mechanisms for transforming the rotation motion in a rectilinear one (less often, the mechanisms based on the use of threaded surfaces and that transform the rectilinear motion in a rotation one could be also met), as fixing elements or to achieve special objectives. If the surface on which the threaded could be placed is considered, one could find that there are cylindrical threads, conical threads, threads developed on a hemispherical surface etc. In the case of cylindrical threads, the main dimensional elements are the external, average and internal diameters and the pitch. The average diameter and the pitch define the inclination angle of the spiral.

There also other criteria applied to classify the threaded surfaces. Thus, one could take into consideration the profile that must be moved along the spiral, the surface on which the thread is placed, the position of this surface (internal or external), the direction of winding, the pitch size, the measuring system, the number of distinct thread spirals etc.

Usually, the threaded surfaces could be obtained by various machining technics $[1,2]$. For example, the threaded surfaces could be obtained by using taps, dies, turning tools, milling tools etc. To improve the quality of the threaded surfaces, techniques such are the grinding and/or lapping could be applied. When the problem of the threaded surfaces

\footnotetext{
* Corresponding author: oanad@tcm.tuiasi.ro
} 
quality is approached, the errors of the main dimensions that characterize the threaded surface could be considered. In some cases, the error of thread flank could be analyzed.

The ways applied to ensure the threaded surfaces accuracy and the influence of some factors on the accuracy of threaded surfaces were sometimes objectives of the scientific research. Thus, Mutambi and Yu investigated the possibilities of using a computer-aided measurement in the case of the external metric screw threads. They highlighted the additional information that could be obtained by means of digital cameras to investigate the detail aspects of the threaded surfaces [3]. Koleva et al. directed their research effort on the study of possibilities to compensate the errors generated by the mechanical deformation when machining threaded surfaces [4]. Their method is based on the inspection of the machined thread profile by means of a workpiece-cutting tool touch probe subsystem.

Aspects concerning the machining system capability to ensure the technological quality of the metric screw thread were studied by Zawada-Tomkiewicz and Wierucka [5]. They developed a measuring system to evaluate some characteristics of a threaded surface, by means of an optical apparatus. They took into consideration the use of the proposed system to highlight the influence of the technological factors on the product quality.

Some mathematical and experimental models corresponding to the obtaining of the threaded surfaces were presented in [1]. Various analyses of the threading processes accuracy were developed in books of machine manufacturing technology [1,6]. A solution for a device intended to be used to evaluate the threading accuracy was proposed in [7].

Within this paper, the results of a theoretical analysis of some factors able to affect the flank error in the case of obtaining threaded surfaces on lathes are discussed.

\section{Initial conditions}

To obtain a threaded cylindrical surface on a lathe, usually the workpiece is rotated, and the cutting tool achieves a movement along the rotation axis as it can be seen in Figure 1. In the case of external metric threads, the cutting tool has to materialize a profile corresponding in principle to an equilateral triangle. To ensure a good machining accuracy, the equilateral triangle must be placed in the workpiece axial plane that, in the case of the universal lathes, is a horizontal plane. Any error of the equilateral triangle correct theoretical shape and position could generate a change of the thread flank profile. For example, if

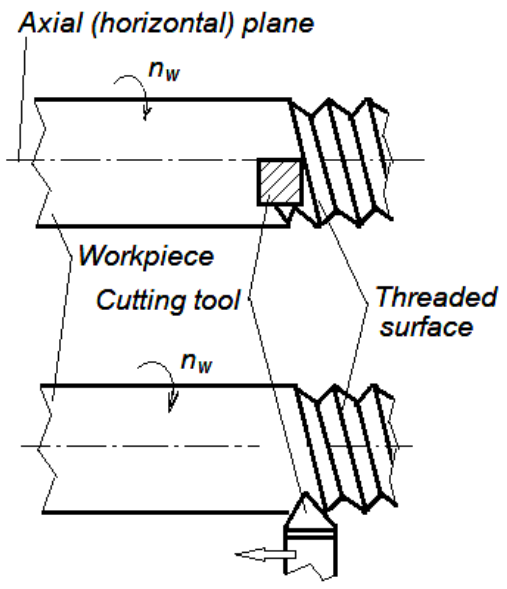

Fig. 1. Obtaining the threaded surface on a lathe, when the turning tool edges must be placed in the horizontal plane of the workpiece axis. under the action of the cutting forces the theoretical equilateral triangle arrives under the horizontal plane, a machining error could appear and this could affect the correct functioning of the threaded surface. If the deviation of the theoretical equilateral triangle from the correct position is low, the machining error could be neglected. For such reasons, the evaluation of the theoretical flank error could present interest from the point of view of the thread accuracy when the threaded surface is obtained by means of the turning tools.

Practically, two sides of the equilateral triangle must be materialized by the two active edges of 


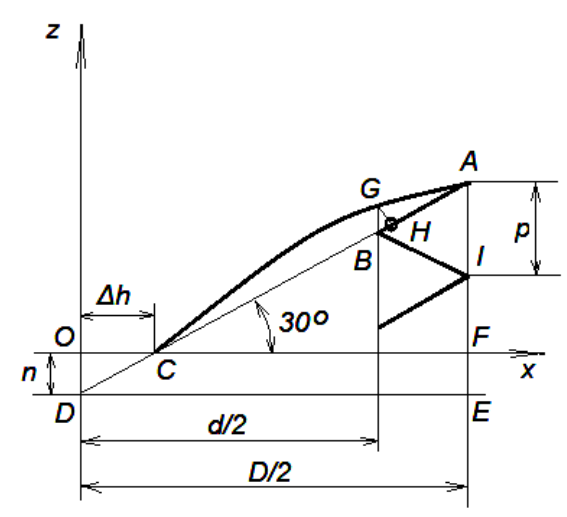

Fig. 2. Details in an axial section through the cylindrical threaded surface.

the turning tool. In accordance with the above mentioned considerations, these two turning tool edges must be placed in the horizontal plane of the workpiece axis, but, for various reasons, it is possible that their position is other than that wanted.

\section{Theoretical flank error}

In Figure 2, an axial section through the cylindrical threaded surface corresponding the metric thread $M 8$ is considered.

In a first approximation, if there is not a longitudinal movement of the turning tool but a transversal movement exists, one could suppose that the active cutting edge generates a conical surface. Since there is not the axial movement of the turning tool, in the horizontal axial plane $x O z$, the segment $A B$ could correspond to the thread flank and it is a generatrix of the conical surface thus generated. The segment $A B$ is a part of the segment AD that could generate the conical surface. This segment has an inclination of 30 degrees to the workpiece rotation axis.

If the tool corner is placed over or under the axial horizontal plane at a distance $\Delta h$, a hyperbolic surface will be generated instead of the conical one. In the horizontal axial plane $x O z$, a hyperbolic curve appears between the points $A$ and $C$, instead of the rectilinear line segment $A C$. Thus, there is also a hyperbole arc $A G$ in the zone delimited by the minor diameter (point $B$ ) and major diameter (point $A$ ). In figure 2, the indicated points have the following significances: $A$ and $I$ - points corresponding to the thread corners, placed on the major diameter; $B$ - point that corresponds to the thread bottom; $C$ - point found on the segment $A D$, at a distance $\Delta h$ from the rotation axis $O z ; D$ - point obtained by the intersection of the right line corresponding to the correct thread flank with the rotation axis $O z ; E$ - point found on a line parallel to the horizontal axis $O x$ that passes through the point $D$; the point $\mathrm{E}$ is found on the cylinder corresponding to the major diameter of the threaded surface; $F$-projection of the point $A$ on the horizontal semi axis $O x ; G$ - point obtained by the intersection of the vertical line that passes through the point $B$ and that intersects the hyperbolic curve; $H$ - point of intersection of the line that passes through the point $G$ and is perpendicular on the segment $A B$. The segment $G H$ corresponds to the thread flank profile error. The distance $n$ is equal to the line segment $O D$ and represents the ordinate of the point in which the line corresponding to the thread intersects the rotation axis $O z$.

If the thread flank profile $\varepsilon_{p z}$ is evaluated along the $O z$ axis, the following relation could be used:

$$
\varepsilon_{p z}=|G B|=z_{G}-z_{B}
$$

Where $G$ is placed on the arc of hyperbole and $B$ is placed on the string of the hyperbola arch.

In accordance with [5], for a similar situation, the following relation corresponds to the hyperbole:

$$
\frac{x^{2}}{\Delta h^{2}}-\frac{z^{2}}{b^{2}}=1
$$


In which the value of the hyperbole semi-axis $b$ is not known. To determine the expression of $b$, the hyperbole relation valid for the point $A$ could be written:

$$
\frac{x_{A}{ }^{2}}{\Delta h^{2}}-\frac{z_{A}{ }^{2}}{b^{2}}=1 .
$$

In the last equation, one could consider:

$$
\begin{aligned}
& x_{A}=|\overline{D E}|=\frac{D}{2} \\
& z=|\overrightarrow{A E}|-|\overrightarrow{F E}|=|\overrightarrow{A E}|-n .
\end{aligned}
$$

From the triangle $D O C$, one could write:

$$
n=\Delta h \cdot \operatorname{tg} 30^{\circ}
$$

And, consecutively:

$$
z=|\overline{A E}|-n=\frac{D}{2} \cdot \operatorname{tg} 30^{\circ}-n .
$$

If in the relation (3), the symbols $x_{A}$ and $z_{A}$ are replaced with their correspondent sizes from the relations (6) an (7), one obtains:

$$
\frac{\left(\frac{D}{2} \operatorname{tg} 30^{\circ}-\Delta h \cdot \operatorname{tg} 30^{\circ}\right)}{b^{2}}=\frac{\left(\frac{D}{2}\right)^{2}}{\Delta h^{2}}-1 .
$$

From this relation, one could write:

$$
b^{2}=\frac{\left(\frac{D}{2} \operatorname{tg} 30^{2}-\Delta h \cdot \operatorname{tg} 30^{\circ}\right)^{2}}{\frac{D^{2}}{4(\Delta h)^{2}}-1}
$$

Or:

$$
b=\frac{\left(\frac{D}{2}-\Delta h\right) \operatorname{tg} 30^{2}}{\sqrt{\frac{D^{2}}{4(\Delta h)^{2}}-1}} .
$$

Taking into consideration the last relation, the hyperbole relation becomes:

$$
\frac{x_{A 2}}{\Delta h^{2}}-\frac{z_{A}{ }^{2}}{\frac{\left(\frac{D}{2}-\Delta h\right)^{2} \operatorname{tg}^{2} 30}{\frac{D^{2}}{4(\Delta h)^{2}}-1}}=1
$$


The relation for the ordinate of a point placed on the hyperbole arc could be extracted from the above written relation:

$$
z_{\text {hyperbole }}=\left(\frac{D}{2}-\Delta h\right)^{2} \operatorname{tg} 30^{\circ} \cdot \sqrt{\frac{\frac{x_{A}{ }^{2}}{\Delta h^{2}}-1}{\frac{D^{2}}{4(\Delta h)^{2}-1}}} .
$$

On the other hand, the relation for the line corresponding to the segment $A B$ is:

$$
z_{\text {right line }}=x \cdot \operatorname{tg} 30^{\circ}-\Delta h \cdot \operatorname{tg} 30^{\circ} .
$$

If we take into consideration the values $\operatorname{tg} 30^{\circ}=0.5774$ and $\Delta h=2 \mathrm{~mm}$, the line equation becomes:

$$
z=0.5775 x-1.1548 \text {. }
$$

To determine the distance between the points placed on the hyperbole arc (corresponding to the thread profile in an axial section through the threaded surface, when there is a certain deviation from the correct position of the cutting tool, during the threading process) and on the right line (corresponding to the correct thread profile) along a direction perpendicular to the line corresponding to the correct profile, firstly the ordinates of the two profiles (correct profile and the hyperbolic profile) were determined and included in the columns nos. 2 and 3 from Table 1, taking into consideration the distance between the major and minor diameter for a metric thread $M 48$ and for $\Delta h=2 \mathrm{~mm}$. In the column no. 4 , the values of the differences $\Delta z$ between the ordinates corresponding to the same abscissa $x$ were mentioned:

$$
\Delta z=z_{\text {hyperbole }}-z_{\text {right line }}
$$

In the column no. 5 from Table 1, the following relation was used to evaluate the profile error:

Table 1. Determination of the profile error.

\begin{tabular}{|c|c|c|c|c|}
\hline $\mathbf{x}$ & $\boldsymbol{z}_{\text {right line }}$ & zhyperbole & Differences $\boldsymbol{\Delta} \boldsymbol{z}, \mathbf{m m}$ & $\begin{array}{c}\text { Thread profile } \\
\text { error } \varepsilon_{t p}, \mathbf{m m}\end{array}$ \\
\hline Column no. 1 & 2 & 3 & 4 & 5 \\
\hline 21.2000 & 11.08608 & 11.209737 & 0.1236574 & 0.107087 \\
\hline 21.4000 & 11.20156 & 11.316435 & 0.1148748 & 0.099482 \\
\hline 21.6000 & 11.31704 & 11.423123 & 0.1060834 & 0.091868 \\
\hline 21.8000 & 11.43252 & 11.529804 & 0.0972835 & 0.084248 \\
\hline 22.0000 & 11.548 & 11.636475 & 0.0884753 & 0.07662 \\
\hline 22.2000 & 11.66348 & 11.743139 & 0.079659 & 0.068985 \\
\hline 22.4000 & 11.77896 & 11.849795 & 0.0708348 & 0.061343 \\
\hline 22.6000 & 11.89444 & 11.956443 & 0.062003 & 0.053695 \\
\hline 22.8000 & 12.00992 & 12.063084 & 0.0531637 & 0.04604 \\
\hline 23.0000 & 12.1254 & 12.169717 & 0.0443171 & 0.038379 \\
\hline 23.2000 & 12.24088 & 12.276344 & 0.0354635 & 0.030711 \\
\hline 23.4000 & 12.35636 & 12.382963 & 0.0266031 & 0.023038 \\
\hline 23.6000 & 12.47184 & 12.489576 & 0.0177359 & 0.015359 \\
\hline 23.8000 & 12.58732 & 12.596182 & 0.0088622 & 0.007675 \\
\hline 24.0000 & 12.7028 & 12.702782 & $-1.79 \mathrm{E}-05$ & $-1.6 \mathrm{E}-05$ \\
\hline
\end{tabular}




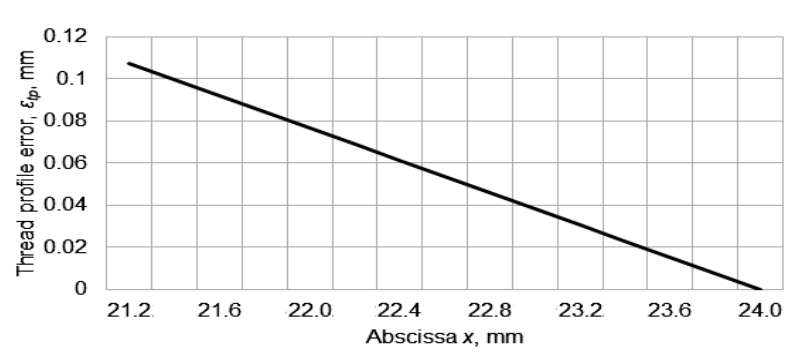

Fig. 3. Variation of the thread profile error $\varepsilon_{t}$ along the thread height in accordance with the equation (16).

$$
\varepsilon_{t p}=\Delta z \cdot \cos 30^{\circ}
$$

The graphical representation from Figure 3 shows the variation of the thread profile error along the distance between the minor and major diameters.

In Table 1, we can see that for the established conditions, the maximum profile thread error is of about $0.1 \mathrm{~mm}$.

\section{Conclusions}

During the obtaining of cylindrical threaded surfaces, one could consider that a certain profile is moved along a cylindrical spiral. In the case of the metric threaded cylindrical surfaces, this profile could be approximated by an equilateral triangle that has a rounded corner. To diminish the value of the thread flank error, the plane corresponding to the equilateral triangle must be placed just in the plane of the axial section through the cylindrical threaded surface. This means that in the case of obtaining the metric thread by turning, the active edges of the cutting tool must be placed in the horizontal axial plane of the rotating workpiece. In this paper, a theoretical model aiming to approximate this error was proposed, for the situation when the corner of the cutting tool is placed under the workpiece horizontal axial plane at a certain distance. For a cylindrical threaded surface M48 and a position of the turning tool corner at a distance of $2 \mathrm{~mm}$ under the horizontal axial plane of the rotating workpiece, the value of the thread profile error was found as being $\varepsilon_{t p} \approx 0.1 \mathrm{~mm}$. In the future, there is the intention to build a device for experimental testing of the theoretical model. The profile error of the flank thread could be measured using a profile projector or a coordinate measuring machine.

\section{References}

1. C. Picoș. Machine manufacturing technology (in Romanian) (Editura Didactică și Pedagogică, Bucharest, 1983)

2. G. Nagîț. Nonconventional technologies (in Romanian) (“Gheorghe Asachi” Technical University, Iași-Romania, 1998)

3. J. Mutambi, Y. Li-jun. Application of digital image analysis method in metric screw thread metrology. Journal of Shanghai University (English Edition) 8(2), 208-212 (2004)

4. S. Koleva, M. Enchev. T. Szecsi. Compensation of the deviations caused by mechanical deformations during machining of threads. Procedia Manufacturing 13, 480486 (2017)

5. A. Zawada-Tomkiewicz. I. Wierucka. A case study in technological quality assurance of a metric screw thread. Measurement 114, 208-217 (2018)

6. O. Pruteanu, A.-E. Stanciu, C. Cărăușu, L. Tăbăcaru. Machining of threads. Theory and experiment (in Romanian) (Publishing House Modtech, Iași-Romania, 2010)

7. L. Slătineanu, M. Radovanovic, M. Coteață, I. Beșliu, O. Dodun, I.Coman, S.-C. Olaru. Requirements in designing a device for experimental investigation of threading accuracy, MATEC Web of Conferences 112, 01005 (2017) 